1. Vazquez-Prado, J., Casas-Gonzalez, P., and Garcia-Sainz, J.A. 2003. G protein-coupled receptor cross-talk: pivotal roles of protein phosphorylation and protein-protein interactions. Cell. Signal. 15:549-557.

2. Drews, J. 1996. Genomic sciences and the medicine of tomorrow. Nat. Biotechnol. 14:1516-1518.

3. Pierce, K.L., Premont, R.T., and Lefkowitz, R.J 2002. Seven-transmembrane receptors. Nat. Rev Mol. Cell Biol. 3:639-650.

4. Hill, S.J. 2006. G-protein-coupled receptors: past, present and future. Br. J. Pharmacol. 147(Suppl. 1): S27-S37.

5. Milligan, G. 2004. G protein-coupled receptor dimerization: function and ligand pharmacology. Mol. Pharmacol. 66:1-7.

6. Prinster, S.C., Hague, C., and Hall, R.A. 2005. Heterodimerization of $\mathrm{G}$ protein-coupled receptors: specificity and functional significance. Pharmacol. Rev. 57:289-298.

7. Bulenger, S., Marullo, S., and Bouvier, M. 2005 Emerging role of homo- and heterodimerization in G-protein-coupled receptor biosynthesis and maturation. Trends Pharmacol. Sci. 26:131-137.

8. Agnati, L.F., Fuxe, K., Zoli, M., Rondanini, C., and Ogren, S.O. 1982. New vistas on synaptic plasticity: the receptor mosaic hypothesis of the engram. Med. Biol. 60:183-190.

9. Mercier, J.F., Salahpour, A., Angers, S., Breit, A., and Bouvier, M. 2002. Quantitative assessment of $\beta 1$ and $\beta 2$-adrenergic receptor homo- and heterodimerization by bioluminescence resonance energy transfer. J. Biol.Chem. 277:44925-44931.

10. Hebert, T.E., et al. 1996. A peptide derived from a $\beta 2$-adrenergic receptor transmembrane domain inhibits both receptor dimerization and activation. J. Biol. Chem. 271:16384-16392.

11. Salahpour, A., et al. 2004. Homodimerization of the $\beta 2$-adrenergic receptor as a prerequisite for cell surface targeting. J. Biol. Chem. 279:33390-33397.

12. Jordan, B.A., Trapaidze, N., Gomes, I., Nivarthi, R., and Devi, L.A. 2001. Oligomerization of opioid receptors with $\beta 2$-adrenergic receptors: a role in trafficking and mitogen-activated protein kinase activation. Proc. Natl. Acad. Sci. U. S. A. 98:343-348.

13. Hague, C., et al. 2006. Heterodimers of $\alpha 1 \mathrm{~B}$ - and $\alpha 1 \mathrm{D}$-adrenergic receptors form a single functional entity. Mol. Pharmacol. 69:45-55.

14. Uberti, M.A., Hague, C., Oller, H., Minneman, K.P., and Hall, R.A. 2005. Heterodimerization with $\beta 2$ adrenergic receptors promotes surface expression and functional activity of $\alpha 1 \mathrm{D}$-adrenergic receptors.
J. Pharmacol. Exp. Ther. 313:16-23.

15. Breit, A., Lagace, M., and Bouvier, M. 2004. Heterooligomerization between $\beta 2$ - and $\beta 3$-adrenergic receptors generates a $\beta$-adrenergic signaling unit with distinct functional properties. J. Biol. Chem. 279:28756-28765.

16. Barki-Harrington, L., Luttrell, L.M., and Rockman, H.A. 2003. Dual inhibition of $\beta$-adrenergic and angiotensin II receptors by a single antagonist: a functional role for receptor-receptor interaction in vivo. Circulation. 108:1611-1618.

17. Grandordy, B.M., Mak, J.C.W., and Barnes, P.J. 1994. Modulation of airway smooth muscle $\beta$-receptor function by a muscarinic agonist. Life Sci. 54:185-191.

18. McGraw, D.W., et al. 2006. Airway smooth muscle prostaglandin- $\mathrm{EP}_{1}$ receptors directly modulate $\beta_{2}$-adrenergic receptors within a unique heterodimeric complex. J. Clin. Invest. 116:1400-1409. doi:10.1172/JCI25840.

19. Barnes, P.J. 1998. Pharmacology of airway smooth muscle. Am. J. Respir. Crit. Care Med. 158:S123-S132.

20. Lee, B., et al. 1998. Influence of the CCR2-V64I polymorphism on human immunodeficiency virus type 1 coreceptor activity and on chemokine receptor function of CCR2b, CCR3, CCR5, and CXCR4. J. Virol. 72:7450-7458.

\title{
An immunologic homunculus for type 1 diabetes
}

\author{
Dirk Homann and George S. Eisenbarth
}

\author{
Barbara Davis Center for Childhood Diabetes, University of Colorado at Denver and Health Sciences Center, Aurora, Colorado, USA.
}

\begin{abstract}
Autoimmune diseases such as the diabetes that develops in NOD mice depend on immunologic recognition of specific autoantigens, but recognition can result in a pathogenic or protective $\mathrm{T}$ cell response. A study by $\mathrm{Du}$ et al. in this issue of the JCI demonstrates that TGF- $\beta$ signaling by T cells recognizing the insulin peptide $B: 9-23$ is essential for such protection and that this inhibitory cytokine functions in both a paracrine and an autocrine manner (see the related article beginning on page 1360). We propose that the insulin peptide B:9-23 and a conserved TCR motif form an "immunologic homunculus" underlying the relatively common targeting of insulin by $T$ cells that, as demonstrated by the study of Du and coworkers, results in a protective $T$ cell response, or diabetes, as shown by other investigators, for related $\mathrm{T}$ cell receptors.
\end{abstract}

There are a limited number of common autoimmune disorders, and some diseases, such as type $1 \mathrm{~A}$ diabetes (immune-mediated type 1 diabetes), both are common and have increased dramatically in incidence over the past 50 years (1). Irun Cohen has advanced the hypothesis that the normal immune repertoire includes a high frequency of lymphocytes that recognize key self-antigens. The relative distribution of lymphocytes specific for various antigens,

Nonstandard abbreviations used: BDC, Barbara Davis Center.

Conflict of interest: The authors have declared that no conflict of interest exists.

Citation for this article: J. Clin. Invest. 116:1212-1215 (2006). doi:10.1172/JCI28506. termed the "immunological homunculus," therefore defines the spectrum of potential autoreactive immune responses (2). The common lack of clinical immunopathology in the presence of a relevant autoreactive lymphocyte repertoire is attributed to active "immune regulation." Conversely, breakdown of such regulatory mechanisms, presciently postulated almost 100 years ago (3), will lead to autoimmune disease. As such tolerance mechanisms are, in part, based on the activity of autoreactive lymphocytes, the increased prevalence of direct consequence of a biased immunologic repertoire (preferential recognition of selected autoantigens), and its particular susceptibility to functional modulation certain autoimmune disorders may be the by environmental and genetic factors. A fundamental question about the pathogenesis of type 1A diabetes is: What is the range of $\mathrm{T}$ cell specificities for islet antigens that modulate induction of pathogenic (destructive) and/or regulatory (protective) $\mathrm{T}$ cell activities; and in particular, can both destructive and regulatory $T$ cells recognize the same antigenic determinant?

\section{T cell specificity, pathogenesis, and prevention of diabetes}

Research over the past few years has identified insulin as a central autoantigen for both the generation of destructive $\mathrm{T}$ cell responses and the therapeutic induction of protective $T$ cell immunity. Until now, the field has lacked $\mathrm{T}$ cell receptor transgenic mice targeting insulin, an important tool in exploring autoimmune pathogenesis. In the current issue of the JCI, Du and coworkers describe the generation of a novel TCR transgenic $(\mathrm{Tg})$ mouse in which the transgenic TCR reacts with a well-defined epitope within the insulin B chain (aa 9-23 or aa 12-25) (4). Although this epitope is recognized by a majority of pathogenic $\mathrm{CD} 4^{+} \mathrm{T}$ cells $(5)$ and may, according to our recent work, constitute the primary autoantigen in the NOD model for type 1 A diabetes (6), the TCR Tg mouse char- 
Table 1

Anti-insulin B:9-23 TCR $\alpha$ chain conservation

\begin{tabular}{|c|c|c|c|c|c|}
\hline $\begin{array}{l}\text { Clone } \\
\text { Yale clone }\end{array}$ & Designation & & $V_{\alpha}$ & $\mathbf{N}$ & $\mathrm{J}_{\alpha}$ \\
\hline $2 \mathrm{H} 6$ & TRAV6S-1 & TRAJ53*01 & VYHCILR & VD & SGGSNYKLTFGKGTLLTVTP \\
\hline \multicolumn{6}{|c|}{ BDC (Barbara Davis Center) clones } \\
\hline $12-4.1$ & TRAV5D-4*04 & TRAJ53*01 & MYFCAAS & GAN & SGGSNYKLTFGKGTLLTVTP \\
\hline $12-4.4$ & TRAV5D-4*04 & TRAJ53*01 & MYFCAAS & A & SGGSNYKLTFGKGTLLTVTP \\
\hline $6-10.14$ & TRAV5D-4*04 & TRAJ53*01 & MYFCAAS & $S R$ & GGSNYKLTFGKGTLLTVTP \\
\hline $4-7.2$ & TRAV5D-4*04 & TRAJ53*01 & MYFCAAS & A N & GGSNYKLTFGKGTLLTVTP \\
\hline $6-4.3$ & TRAV5D-4*04 & TRAJ53*01 & MYFCAAS & A S G & SGGSNYKLTFGKGTLLTVTP \\
\hline 8-1.3(LN) & TRAV5D-4*01 & TRAJ42 & MYFCAAS & $A R G$ & SGGSNYKLTFGKGTKLSVKS \\
\hline $12-3.20$ & TRAV5D-4*04 & TRAJ42 & MYFCAAS & K I & GGSNYKLTFGKGTKLSVKS \\
\hline 8-1.9(LN) & TRAV5D-4*04 & TRAJ42 & MYFCAAS & R P & GGSNYKLTFGKGTKLSVKS \\
\hline 8-1.1(LN) & TRAV5D-4*04 & TRAJ56 & MYFCAAS & K & TGGNNKLTFGQGTVLSVIP \\
\hline 8-1.5(LN) & TRAV5D-4*04 & TRAJ13 & MYFCAAS & $A$ & NSGTYQRFGTGTKLQVVP \\
\hline $12-1.19$ & TRAV13 & TRAJ11 & TYLCAME & R S & SGYNKLTFGKGTVLLVSP \\
\hline $12-2.35$ & TRAV12 & TRAJ23 & LYFCAAI & $Q$ & NYNQGKL I FGQGTKLSIKP \\
\hline $6-6.4$ & TRAV7 & TRAJ57 & LYYCAPN & $Q$ & GGSAKL I FGEGTKLTVSS \\
\hline
\end{tabular}

The $\alpha$ chain of the $2 \mathrm{H} 6$ clone, used to produce the TCR Tg mice described in this issue of the JC/ by Du et al. (4), uses the dominant conserved J $\alpha$ chain (TRAJ53*01). Conserved J $\alpha$ is shown in blue boldface for name and sequence KLTFGKGT. Dominant conserved V $\alpha$ sequences and designation are shown in black boldface.

acterized by Du et al. produces $\mathrm{T}$ cells that spontaneously prevent diabetes. Almost a decade ago, Zekzer et al. described a CD4 ${ }^{+}$ $\mathrm{T}$ cell clone (2H6) derived from pancreatic lymph nodes of NOD mice that recognized the insulin B:9-23 and B:12-25 epitopes, that produced both TGF- $\beta$ and IFN- $\gamma$, that was able to home to islets, and that prevented spontaneous diabetes in NOD mice (7). Of note, the TCR of this $\mathrm{CD}^{+} \mathrm{T}$ cell clone shared a common J $\alpha$ chain motif (KTLFGKGT) found in 3 of 50 germ-line J $\alpha$ sequences (Table 1 ), but used by the majority of pathogenic clones reacting with insulin peptide B:9-23 (8).

Role of TGF- $\beta$ in diabetes protection $\mathrm{Du}$ and coworkers now have engineered a transgenic NOD mouse with the TCR sequences from the protective $2 \mathrm{H} 6$ clone (4). Not only were the TCR Tg mice protected from the development of spontaneous diabetes, but the transfer of $2 \mathrm{H} 6$ TCR Tg cells together with diabetogenic $\mathrm{T}$ cells from NOD mice or a pathogenic $\mathrm{T}$ cell clone (clone name BDC2.5) reacting with an unknown islet antigen protected recipient animals from $\mathrm{BDC} 2.5$-induced diabetes. The capacity of $2 \mathrm{H} 6 \mathrm{~T}$ cells to provide protection in the absence of any further in vitro manipulation is particularly striking as it has been reported that a TCR from anti-B:9-23 pathogenic clones, despite reacting with the same insulin B:9-23 peptide, causes spontaneous dia- betes in Tg mice (9). Thus, TCRs targeting the same insulin epitope can either cause or prevent diabetes.

The study by Du and coworkers (4) has gone further to explore the mechanism by which the TCR Tg T cells mediate protection against the development of diabetes. Although TGF- $\beta$, produced by both the $2 \mathrm{H} 6$ clone (10) and naive TCR Tg 2H6 $\mathrm{T}$ cells (4), is an obvious candidate, the complex functions of this cytokine in Treg biology remain somewhat controversial - in particular the role played by $T$ cell-produced TGF- $\beta$ in autoimmune disease protection (11). By use of cotransfer experiments combining diabetogenic $\mathrm{T}$ cells carrying a dominant-negative TGF$\beta$ receptor II with protective $2 \mathrm{H} 6 \mathrm{~T}$ cells, the authors found that pathogenic $\mathrm{T}$ cells that are unable to respond to TGF- $\beta$ signals cannot be prevented from causing diabetes in recipient mice. Complementary experiments with injection into test mice of TGF- $\beta$-insensitive $2 \mathrm{H} 6 \mathrm{~T}$ cells and normal pathogenic $\mathrm{T}$ cells also failed to protect the recipient mice from diabetes, indicating that Treg-produced TGF- $\beta$ may act in both a paracrine fashion (modulating destructive activity of diabetogenic cells) and an autocrine fashion (preserving their regulatory activity). Although these experiments clearly suggest an important role for TGF- $\beta$ production by Tregs, definitive proof will have to come from future experiments using
$T$ cell-specific, conditional deletion of TGF- $\beta 1$, the predominant TGF- $\beta$ species produced by $\mathrm{T}$ cells.

\section{Tregs and antigen specificity}

What are the lessons to be drawn from this animal study regarding the pathogenesis and prevention of human type 1 diabetes? The current study (4) documents the existence of antigen-specific Tregs and demonstrates that such cells need not conform to the natural Treg phenotype (i.e., they are not $\mathrm{CD}_{25} 5^{+}$, nor do they express the signature transcription factor FoxP3). Perhaps more importantly, the study indicates that epitope specificity per se does not predict the nature of autoreactive immune responses, since protective and pathogenic $\mathrm{T}$ cells can recognize similar autoantigenic epitopes, if not the same autoantigenic epitope. Therefore, concerns are warranted that antigen-specific immunotherapies could skew the balance between pathogenic and protective $\mathrm{T}$ cell immunity toward pathogenicity. A clinical trial of an altered peptide ligand of insulin B:9-23 is currently under way, and the results of this trial, as well as a trial of insulin B chain administered in incomplete Freund's adjuvant, are awaited with interest (12). The study by Du and colleagues (4) provides a few clues that may explain the "protective phenotype" of the transgenic T cells: the $2 \mathrm{H} 6 \mathrm{~T}$ cells do not exhibit a genuine naive phenotype and are apparently cross-reactive with an 
undefined antigen other than the insulin $\mathrm{B}$ chain. In addition, $2 \mathrm{H} 6 \mathrm{~T}$ cells recovered from pancreatic lymph nodes and, to a lesser extent, the spleen, but not from bone marrow or thymus, demonstrated efficient protective capacities. Thus it is conceivable that intermittent or prolonged exposure to "persisting" antigens favors the generation of regulatory rather than pathogenic $T$ cell reactivity (13). The availability of TCR Tg NOD mouse models that maintain autoreactive $T$ cells in the presence versus the absence of autoantigen should allow for direct testing of this hypothesis (14).

\section{Insulin as a potential primary autoantigen in type 1 diabetes}

Insulin is obviously not the only islet peptide recognized by anti-islet-specific $\mathrm{CD}^{+}$and $\mathrm{CD}^{+}{ }^{+} \mathrm{T}$ cells in the NOD mouse (15) but is, to date, the only antigen that, upon deletion or alterations of its immunogenic properties, dramatically accelerates or prevents diabetes $(6,16-20)$. Reactivity of T cells to other islet antigens such as glutamic acid decarboxylase and the $\beta$ cell-specific protein islet-specific glucose6-phosphatase catalytic subunit-related protein (IGRP) (21) has been described, and the precise identities of some targets remain to be determined. Nevertheless, we believe it is likely that specific properties of the insulin B chain, and the relatively simple, low-stringency TCR motif implicated in recognizing the $\mathrm{B}: 9-23$ sequence (Table 1) in the context of appropriate MHC molecules, make this epitope a preferred target for immunomodulation. Although it is much easier to posit a primary autoantigenic epitope for disease activation in an animal model with a single class II MHC molecule (e.g., I-A ${ }^{\mathrm{g} 7}$ ), we suggest that the immunogenicity of insulin and its recognition by autoreactive $\mathrm{T}$ cells may in fact drive the high prevalence of type 1 diabetes in humans.

A remarkable dominance of MHC molecules underlies type 1 diabetes in humans, with the highest-risk class II genotype (DR3-DQ2:DR4-DQ8) making up onethird of individuals who develop the disease versus a population frequency of $2.4 \%$ in Denver, Colorado (22). In addition, HLA molecules such as DQB $1 * 0602$ provide dominant protection from type 1 diabetes in multiple populations $(23,24)$. Nevertheless, there is a different disease risk for each MHC genotype, and though it is possible that only a single peptide epitope will relate to disease with multiple MHC genotypes, this contention remains to be evaluated experimentally. In a recent study of pancreatic lymph nodes from 3 individuals with diabetes, 2 had T cells with marked conservation of their TCRs that reacted with insulin A chain peptide A:1-15 (25). We believe that further study of both the pancreatic lymph nodes and the islets of individuals who develop type 1 diabetes is essential, and if dominant TCRs and target autoantigens similar to those of the NOD mouse can be identified, it will greatly facilitate molecular understanding of the pathogenesis of type 1 diabetes.

\section{Toward targeted immunotherapies: the challenges ahead}

The therapeutic targeting of autoimmune epitopes with high immunogenicity (e.g., insulin B:9-23 in NOD mice) may be conducive to regulatory rather than pathologic $\mathrm{T}$ cell immunity as long as proper regulatory circuits are in place. However, compromised Treg functions will facilitate generation of destructive autoimmune responses, as illustrated, for example, by patients with FoxP3 mutations who suffer from overwhelming autoimmunity, including development of neonatal type 1 diabetes (26). Although important for many aspects of Treg function, FoxP3 expression is not a hallmark of all Tregs, as evidenced by the current study (4) as well as earlier work (27), and other factors such as impaired central tolerance due to mutations of the autoimmune regulator (AIRE) gene that result in widespread autoimmunity (26) or environmentally induced alterations (e.g., resulting in IFN- $\alpha$ induction) contribute to the eventual balance between pathogenic and protective immune responses. The study by Du and coworkers (4) provides important insights toward the understanding of determinants that modulate immunoregulation targeted to a potentially primary autoantigen. In the past few years, we have learned to predict diabetes development in humans and prevent, as well as revert, clinical diabetes in various animal models (28). At present, however, clinical trials for the prevention of diabetes in humans are limited by the lack of adequate assays that monitor specific $T$ cell activities associated with therapeutic efficacy or progression of disease (29). The development of such assays is essential for the design and monitoring of effective clinical trials, and it will be of interest to use reagents and derivatives such as the $2 \mathrm{H} 6$ TCR Tg mice (combined with pathogenic $T$ cells recognizing the same and other antigens) to assist in the development of such assays.

\section{Acknowledgments}

This work was supported by the NIH (grants AG026518-01 and DK55969-08), the American Diabetes Association, the Juvenile Diabetes Research Foundation International, and the Children's Diabetes Foundation.

Address correspondence to: George S. Eisenbarth, Barbara Davis Center for Childhood Diabetes, MS B140, Box 6511, 1775 North Ursula Street, Aurora, Colorado 80010, USA. Phone: (303) 724-6847; Fax: (303) 724-6839; E-mail: george.eisenbarth@ uchsc.edu.

1. Gale, E.A. 2002. The rise of childhood type 1 diabetes in the 20th century. Diabetes. 51:3353-3361.

2. Cohen, I.R. 1992. The cognitive paradigm and the immunological homunculus. Immunol Today. 13:490-494.

3. Römer, P., and Gebb, H. 1912. Beiträge zur Frage der Anaphylaxie durch Linseneiweiss und Eiweiss aus andern Geweben des Auges. Albrecht Von Graefes Arch. Ophthalmol. 81:367-402.

4. Du, W., et al. 2006. TGF- $\beta$ signaling is required for the function of insulin-reactive $T$ regulatory cells. J. Clin. Invest. 116:1360-1370. doi:10.1172/JCI27030.

5. Daniel, D., Gill, R.G., Schloot, N., and Wegmann, D. 1995. Epitope specificity, cytokine production profile and diabetogenic activity of insulin-specific $\mathrm{T}$ cell clones isolated from NOD mice. Eur. J. Immunol. 25:1056-1062.

6. Nakayama, M., et al. 2005. Prime role for an insulin epitope in the development of type 1 diabetes in NOD mice. Nature. 435:220-223.

7. Zekzer, D., et al. 1997. Inhibition of diabetes by an insulin-reactive CD4 T-cell clone in the nonobese diabetic mouse. Diabetes. 46:1124-1132.

8. Simone, E., et al. 1997. Cell receptor restriction of diabetogenic autoimmune NOD T cells. Proc. Natl. Acad. Sci. U. S. A. 94:2518-2521.

9. Jasinski, J., et al. 2005. BDC12-4.1 (anti-insulin B:9-23) T cell receptor transgenic mice are lymphopenic but entrain early insulitis and can cause early diabetes modified by insulin 2 gene expression [abstract]. Paper presented at the 2005 Annual Meeting of the Federation of Clinical Immunology Societies, Inc. May 12-16. Boston, Massachusetts, USA.

10. Zekzer, D., Wong, F., von Grafenstein, H., and Sherwin, R.S. 1996. An insulin-reactive T cell clone prevents IDDM in NOD mice by blocking homing of diabetogenic splenocytes to the islet [abstract]. Diabetes. 45(Suppl. 2):189A.

11. Li, M.O., Wan, Y.Y., Sanjabi, S., Robertson, A.K., and Flavell, R.A. 2006. Transforming growth factor-beta regulation of immune responses. Annu. Rev. Immunol. 24:99-146.

12. Alleva, D.G., et al. 2002. Immunological characterization and therapeutic activity of an alteredpeptide ligand, NBI-6024, based on the immunodominant type 1 diabetes autoantigen insulin B-chain (9-23) peptide. Diabetes. 51:2126-2134.

13. Homann, D., and von Herrath, M. 2005. "Natural" and "induced" regulatory T cells: purpose and problems associated with an emerging distinction. In Regulatory T cells in inflammation. L.S. Taams, A.N. Akbar, and M.H. Wauben, editors. Birkhäuser Verlag. Basel, Switzerland. 19-38. 
14. Pauza, M.E., et al. 2004. T-cell receptor transgenic response to an endogenous polymorphic autoantigen determines susceptibility to diabetes. Diabetes. 53:978-988.

15. Lieberman, S.M., and DiLorenzo, T.P. 2003. A comprehensive guide to antibody and T-cell responses in type 1 diabetes. Tissue Antigens. 62:359-377.

16. Moriyama, H., et al. 2003. Evidence for a primary islet autoantigen (preproinsulin 1) for insulitis and diabetes in the nonobese diabetic mouse. Proc. Natl. Acad. Sci. U. S. A. 100:10376-10381.

17. Thebault-Baumont, K., et al. 2003. Acceleration of type 1 diabetes mellitus in proinsulin 2-deficient NOD mice. J. Clin. Invest. 111:851-857. doi:10.1172/ JCI200316584.

18. Jaeckel, E., Lipes, M.A., and von Boehmer, H. 2004. Recessive tolerance to preproinsulin 2 reduces but does not abolish type 1 diabetes. Nat. Immunol. 5:1028-1035

19. Kubosaki, A., et al. 2004. Targeted disruption of the IA-2beta gene causes glucose intolerance and impairs insulin secretion but does not prevent the development of diabetes in NOD mice. Diabetes. 53:1684-1691.

20. Kubosaki, A., Miura, J., and Notkins, A.L. 2004. IA2 is not required for the development of diabetes in NOD mice. Diabetologia. 47:149-150.

21. Lieberman, S.M., et al. 2003. Identification of the beta cell antigen targeted by a prevalent population of pathogenic CD8+ T cells in autoimmune diabetes. Proc. Natl. Acad. Sci. U. S. A. 100:8384-8388.

22. Robles, D.T., et al. 2002. Millennium award recipient contribution. Identification of children with early onset and high incidence of anti-islet autoantibodies. Clin. Immunol. 102:217-224.

23. Redondo, M.J., et al. 2000. DR and DQ associated protection from type 1 diabetes: comparison of DRB1*1401 and DQA1*0102-DQB1*0602. J. Clin. Endocrinol. Metab. 85:3793-3797.

24. Valdes, A.M., et al. 2005. D6S265*15 marks a DRB1*15, DQB1*0602 haplotype associated with attenuated protection from type 1 diabetes mellitus. Diabetologia. 48:2540-2543.

25. Kent, S.C., et al. 2005. Expanded T cells from pan- creatic lymph nodes of type 1 diabetic subjects recognize an insulin epitope. Nature. 435:224-228.

26. Chen, Z., Benoist, C., and Mathis, D. 2005. How defects in central tolerance impinge on a deficiency in regulatory T cells. Proc. Natl. Acad. Sci. U. S. A. 102:14735-14740.

27. Vieira, P.L., et al. 2004. IL-10-secreting regulatory T cells do not express Foxp 3 but have comparable regulatory function to naturally occurring CD4+CD25+ regulatory T cells. J. Immunol. 172:5986-5993.

28. Eisenbarth, G.S. 2005. Prediction of type 1 diabetes: the natural history of the prediabetic period. In Type 1 diabetes: molecular, cellular and clinical immunology. Barbara Davis Center for Childhood Diabetes. http://www.uchsc.edu/misc/diabetes/ oxch11.html.

29. Roep, B.O., et al. 1999. Autoreactive T cell responses in insulin-dependent (type 1) diabetes mellitus. Report of the first international workshop for standardization of T cell assays. J. Autoimmun. 13:267-282.

\title{
Bypassing complement: evolutionary lessons and future implications
}

\author{
John P. Atkinson ${ }^{1}$ and Michael M. Frank ${ }^{2}$ \\ 1Department of Medicine, Division of Rheumatology, Washington University School of Medicine, St. Louis, Missouri, USA \\ 2Department of Pediatrics, Immunology and Medicine, Duke University Medical Center, Durham, North Carolina, USA.
}

Lectins like mannan-binding protein are part of the innate immune system. They circulate in association with serine proteases. Upon binding oligosaccharides, they activate the complement cascade analogous to the more familiar but evolutionarily more recent classical pathway, which is triggered by antibody binding to antigen. In this issue of the JCI, Selander et al. developed a sensitive and specific ELISA employing Salmonella-specific sugars to assess the activity of the lectin pathway of complement activation (see the related article beginning on page 1425). This more physiologic assay system allowed the investigators to rigorously define the requirements for lectin pathway activation. Furthermore, they uncovered an unsuspected means for this pathway to reach the desired critical step of activation of the opsonin C3. These types of functional assays will eventually replace the more laborious, less physiologic, and less informative approaches currently in use to monitor complement activation.

Innate immunity suffices for most organisms on this planet. Antibodies and lymphocytes did not appear on the evolutionary scene until cartilaginous fish and sharks. The complement system is a major player in this innate immunity. The initial half of this proteolytic complement cascade accomplishes 2 goals: first, to deposit opsonic fragments derived from comple-

Nonstandard abbreviations used: MASP, MBL-associated serine protease; MBL, mannan-binding lectin.

Conflict of interest: The authors have declared that no conflict of interest exists.

Citation for this article: $J$. Clin. Invest. 116:1215-1218 (2006). doi:10.1172/JCI28622. ment components 3 and 4 (C3 and $\mathrm{C} 4$, respectively) on a target, and second, to initiate the inflammatory response through release of the $\mathrm{C} 3 \mathrm{a}$ anaphylatoxin. These 2 events occur in parallel and within minutes, making complement activation an early (if not the earliest) part of the host defense response to a microbe. Three activation pathways - classical (Figure 1), lectin (Figure 2), and alternative (Figure 3) - have been elucidated. They join at the step of C3 cleavage. Common to each pathway is the terminal half of the system, the membrane attack complex. This lytic system is initiated with the cleavage of C5 to $\mathrm{C} 5 \mathrm{a}$, another potent anaphylatoxin, and
C5b, which combines with $\mathrm{C} 6, \mathrm{C} 7, \mathrm{C} 8$, and C9 to form a pore or channel in the pathogen's cell membrane. Deficiencies in complement components predispose to infection and autoimmunity. While it is rather straightforward to account for the former, it has not been so easy for immunologists to explain the latter.

The complement system is activated by lectins, representing a preantibody means of recognizing non-self by targeting the distinct sugar profiles of microorganisms (Figure 2). Not surprisingly, parts of the lectin complement cascade were then "borrowed" by the adaptive immune system to form the antibody-triggered classi-

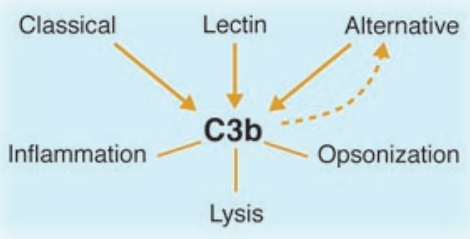

Figure 1

Three pathways of complement activation. Deposition of clusters of C3b on a target is the primary goal. The alternative pathway also serves as a feedback loop such that C3b deposition can be amplified (shown by broken line). 